\title{
La qualité en oncologie
}

\section{Jürg Nadig}

Dr med., président de la Société suisse d'oncologie médicale (SSMO)

\author{
Le projet de Loi fédérale sur l'enregistrement des maladies oncologiques (LEMO) est \\ actuellement soumis au Parlement. Même si les registres ne répondent pas à toutes \\ les questions, ils méritent le soutien du corps médical et créent les bases légales \\ permettant de répondre aux questions de la qualité des soins.
}

Le projet de Loi fédérale sur l'enregistrement des maladies oncologiques (LEMO) est actuellement soumis au Parlement. Ce n'est pas une mince affaire que de réunir plusieurs objectifs dans une même loi. Mais contrôler la qualité et les coûts de santé doit passer par les éléments essentiels que sont la multidisciplinarité du processus thérapeutique et le projet de LEMO fédérale [1, 2]. C'est en ce sens que la LEMO doit aussi bien intégrer les données épidémiologiques que les données thérapeutiques mises à disposition des sociétés médicales pour leur registre de qualité [3]. A titre d'exemple, c'est sur la base de ces données que l'on pourrait vérifier si en Suisse, comme aux Etats-Unis, les patients bénéficient d'un meilleur traitement dans les institutions de recherche. Ou, alternativement, si cette observation n'est pas valable en Suisse du fait, par exemple, que des moyens financiers adéquats permettent de proposer une bonne qualité de traitement, même dans les institutions dépourvues d'activités de recherches.

\section{Disposer d'informations détaillées}

Correspondance:

Dr Jürg Nadig, MAE

Bannhaldenstrasse 7

CH-8180 Bülach

juerg.nadig[at]hin.ch
Alors que le processus thérapeutique révèle ce qui tient des standards à minima, les registres de données peuvent indiquer combien de patients reçoivent un

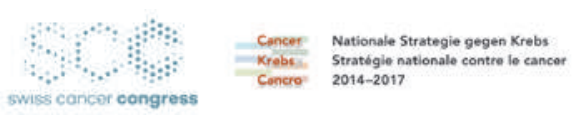

\section{$2^{\mathrm{e}}$ Congrès suisse du cancer}

Le 27 août 2015 se déroulera à I'Université de Fribourg le $2^{\mathrm{e}}$ Congrès suisse du cancer. Ce congrès a été créé l'an dernier par les prestataires de soins en oncologie. Cette année, il sera organisé conjointement avec des acteurs de la Stratégie nationale contre le cancer. Le Congrès suisse du cancer se veut un think tank, une rencontre de travail annuelle et un forum destiné à faire progresser en continu tous les champs d'intervention de l'oncologie: prévention, traitement, prise en charge et recherche. Cette manifestation vise enfin à favoriser la mise en réseau et les échanges interdisciplinaires et interprofessionnels entre les divers prestataires de soins et les institutions. Cette année, le congrès aura pour sujet la qualité en ocologie. Il s'articulera sur des ateliers parallèles qui permettront aux participants d'approfondir une thématique sous l'angle de la qualité et d'en discuter. Une mise à jour concernant la Stratégie nationale contre le cancer est également prévue.

Sont invités tous les médecins, soignants et professionnels qui interviennent dans le parcours des patients atteints de cancer.

Informations et inscription: www.swisscancercongress.ch traitement basé sur la preuve (donc conforme aux guidelines) et dans quelle mesure celui-ci influence leur survie hors études cliniques [4]. Pour cela, il est indispensable d'alimenter les registres cantonaux des tumeurs avec des informations détaillant le diagnostic et les traitements réalisés. Pour que ces informations soient traitées dans les meilleures conditions, il est essentiel que leur mode de saisie soit aisé et que les flux de données soient clairement définis. Les données doivent également être recueillies pendant toute la durée de suivi et leur conservation doit être suffisamment longue, puisque l'évolution de la maladie dépend de séquences thérapeutiques étalées dans le temps.

\section{Bien des questions encore en suspens}

Comment mettre en œuvre un traitement «conforme aux guidelines» quand les patients sont toujours plus nombreux à présenter une polymorbidité et quand ils sont aussi invités à participer aux décisions thérapeutiques (shared decision making)? Les registres sont-ils en mesure de répondre à ce type de question? Et quelle situation serait à valoriser: atteindre l'objectif ou traiter selon les guidelines? Même si les registres ne peuvent répondre à ces questions, ce projet de loi mérite le soutien du corps médical. Il pose en effet certaines bases juridiques dans le domaine de l'oncologie. Il instaure notamment la mise en place d'un robuste corpus de données qui permettra de répondre à bien des questions relatives à la qualité des soins et sur lequel les sociétés médicales s'appuieront pour leurs projets en matière de qualité en oncologie.

1 Bosshard C, Meyer-Nikolic VA, Nadig J, Pfisterer J. Projet-pilote «Trajectoire intersectorielle des patients atteints du cancer du côlon». Bull Méd Suisses. 2014;95(31-32):1136-7.

2 www.bag.admin.ch/themen/gesundheitspolitik/10374/index. html?lang=fr\&download=NHzLpZeg7t,lnp6IONTU04212Z6ln1ae2I Zn4Z2qZpnO2Yuq2Z6gpJCMdYR_gWym162epYbg2c_JjKbNoKSn6A--

3 Nadig J, Gruber G. Qualitätsregister der Fachgesellschaften als Beitrag zum NKP II. Schweizer Krebsbulletin 32. 71; 2013.

4 Nadig J, Gähler E. Guidelines taugen nicht zu Wirtschaftlichkeitsverfahren. Bull Méd Suisses. 2011;92(43):1660-2. 